\title{
The Role of Video-Assisted E-Learning in Intrauterine Contraceptive Device Insertion Training
}

Ng Lai Peng, MBBS, MMed | Eileen Koh Yi Ling, Bsc

PRiMER. 2021;5:5.

Published: 1/28/2021 | DOI: 10.22454/PRiMER.2021.298435

\section{Abstract}

Introduction: Intrauterine contraceptive device (IUCD) insertion is an important procedural skill for primary care physicians. Procedural skill training can be challenging. E-learning has been rapidly employed in medical education. The purpose of this study was to evaluate the role of a video-assisted e-learning module in knowledge and skill transfer for IUCD insertion training.

Methods: We used a quasi-experimental, one-group, pre/posttest study design. Thirty-one primary care doctors (family medicine residents and new medial officers) at a primary care training center participated in the study. All participants assessed the e-learning module at the training center. We performed on-site pre/posttests of knowledge and procedural steps (posttest only). We obtained satisfaction with e-learning and perceived confidence level with an anonymous questionnaire survey.

Results: There was a significant increase in the knowledge test scores from a median interquartile range score of 12 (10-15) to 18 (17-19) post-e-learning $(P<.01)$. All participants attained the minimum passing score of 15 of 20 (75\%) post-e-learning. However, only 20 of $31(64.5 \%)$ participants met the minimum passing standard for procedural step test post-e-learning. Ninety-three percent of the participants reported satisfaction with the e-learning course. The median score for confidence level in performing the procedure increased significantly after e-learning.

Conclusion: The e-learning module may be an effective way of delivering instructional content in procedural training. It provides time and location flexibility and is useful for pretraining. Psychomotor skills for clinical procedures, however, may be difficult to acquire by e-learning alone. It must be supplemented with deliberate practice and hands-on learning in simulation workshops.

\section{Introduction}

The intrauterine contraceptive device (IUCD) is an effective and safe long-acting reversible contraceptive method. ${ }^{1,2}$ It is an important procedural competency skill for primary care physicians. ${ }^{3-5}$

Procedural skill training has been challenging. ${ }^{6,7}$ Well-designed, high-quality e-learning may assist in filling up the gap in procedural skill training in family medicine. ${ }^{6}$ 
Computer-assisted instruction has been previously explored in clinical skill training. ${ }^{8}$ Instructional video has been shown to supplement clinical teaching for basic clinical procedures such as venipuncture ${ }^{9}$ and surgical skill training. ${ }^{10,11}$ Videos enable better visualization of the clinical procedure and allow learners to be better prepared for clinical psychomotor skills. ${ }^{12}$ The repetitive nature of video-module instruction allows learners to selectively review the steps. ${ }^{13}$ However, there is a paucity of studies on the effectiveness of video-assisted e-learning in clinical procedural skill training in family medicine.

A study comparing video module with the traditional form of instruction on IUCD insertion suggests that the video module is just as effective in providing procedural skill instruction for IUCD insertion. ${ }^{3}$ We developed an e-learning module on IUCD insertion for doctors in family practice. We used the Nova T IUCD device for the study as it involves more steps in its insertion. We studied the role of video-assisted e-learning in IUCD insertion training. We explored its capability in procedural knowledge and skill transfer.

\section{Methods}

\section{Video-Assisted E-Learning Module}

We created a 20-minute e-learning module using Mayer's multimedia principles ${ }^{14}$ with interactive components incorporated. ${ }^{15}$ The content of the module was designed around key components described for prepatient training for procedural skills. ${ }^{2,16-19}$ These included cognitive knowledge on the procedure, eligibility criteria, indications, contraindications, complications, pre- and postprocedural counseling, steps, and fundamental elements of the procedure. ${ }^{17-19}$ We included animated instructional video with detailed illustration to allow learners to visualize each step of the clinical procedure.

\section{Setting}

We conducted the study at a primary care training center. Participants were family medicine residents and medical officers new to the public primary care polyclinics. All 31 participants invited gave informed consent for the study.

\section{Study Design and Intervention}

We conducted a quasi-experimental, one-group, pre/posttest study to assess the transfer of content knowledge and procedural steps (posttest only) with e-learning. We assessed satisfaction with e-learning and perceived confidence level in procedure using an anonymous questionnaire survey. ${ }^{20}$

We assessed pretest knowledge using 20-item multiple-choice questions (MCQ). ${ }^{21}$ Participants engaged in individual e-learning sessions onsite. This was followed by a posttest assessment with the same set of MCQ questions.

Participants were then required to demonstrate the steps of insertion of the Nova T IUCD device on a plastic model individually, while being video recorded. The anonymized recordings were scored by two independent raters using a procedural step checklist. ${ }^{22}$

\section{Tools for Outcome Assessment}

We used absolute standard setting ${ }^{23-25}$ to determine the minimum passing score for the knowledge test, set at $75 \%$ ( 15 out of 20 ). We used conjunctive standard setting ${ }^{23-25}$ for the procedural step test; two critical items must be performed besides meeting the minimum total score of 14 out of 18 to meet the minimum passing standard (MPS) of the procedural step test. The MPS of the tests was determined prior to the study by a team of three family physicians with experience in procedural training. 


\section{Statistical Analysis}

We used Wilcoxon signed rank test to compare the pre/post-e-learning scores of the knowledge test. We used Mann-Whitney $U$ test to assess the demographics and the satisfaction score on e-learning. We used $\chi^{2}$ test to compare the procedural step test result and demographics, while we used Mann Whitney $U$ test to predict the procedural step test result based on post-e-learning knowledge test score and self-rated confidence level. We considered a $P$ value of less than .05 significant.

We estimated procedural step test score reliability by calculating interrater reliability using 2-way mixed intraclass coefficient (ICC) for the total score and kappa coefficient for the attainment of MPS.

We submitted this study to Singhealth Centralised Institutional Review Board CIRB (Ref: 2016/3094). A formal review was determined to be not required.

\section{Results}

All 31 participants' completion of the procedure were video recorded, and all completed the pre/post knowledge test questions. Two participants did not complete the online satisfaction questionnaire survey, and the gender of one of the participants was not captured online (Table 1).

\section{Knowledge Test}

There was a significant increase in the knowledge scores from a median interquartile range (IQR) score of 12 $(10-15)$ to 18 (17-19) post-e-learning $(P<.01)$. All participants attained the minimum passing score of 15 of 20 post-e-learning (Figure 1).

\section{Procedural Step Test}

Twenty of 31 (64.5\%) participants met the minimum passing standard (MPS) for procedural step test poste-learning (Figure 2). The interrater reliability for the attainment of MPS for the procedural step test had a perfect kappa coefficient of $1.00(P<.001)$ between the two raters. The ICC for the total score was 0.85 $(P<.001)$.

There was no difference in the median MCQ scores for participants who passed or failed the procedural step test. There was no correlation between the post-e-learning knowledge score and the procedural step test score.

\section{Satisfaction}

Ninety-three percent of participants agreed/strongly agreed that they were satisfied with the e-learning course (Figure 3). The median score for confidence level in performing the procedure increased from 2 out of 10 (IQR $1-4.75)$ to 7 (IQR 5.25-8; $P<.01)$ post-e-learning.

We found no correlation between the satisfaction level, knowledge test score, procedural step test score, and gender or age.

\section{Conclusion}

The e-learning module may be an effective way of delivering instructional content in procedural training. It allows consistent content to be delivered with time and location flexibility. However, there is limited ability for transfer of psychomotor skill in clinical procedural training. In our study, 35.5\% of learners failed the procedural step test post-e-learning. This suggests that psychomotor skills may not be acquired by e-learning alone for 
many learners.

The psychomotor phase in procedural skills training requires repeated physical practice with correction and reinforcement. ${ }^{26,27}$ This may be achieved with simulation-based training that allows repetitive practice and direct feedback for mastery of skills. ${ }^{2}$

Our study provides realistic information on the role of e-learning in procedural skill training. It may be an effective way to deliver content knowledge in procedural skill training. Our learners reported great satisfaction and improved confidence level post-e-learning. Psychomotor skills, however, must be supplemented with deliberate practice and hands-on learning.

Interestingly, we found no correlation between the post-e-learning knowledge test score and the procedural step skill test score. This suggests differences between knowledge and psychomotor skills acquisition.

A limitation of our study is that it was a pre/posttest performance study with no comparative group on traditional didactic teaching. The posttest performance was conducted immediately post-e-learning, and long term retention of knowledge was also not assessed.

In these unprecedented times, where there is limited opportunity for face-to-face teaching, preprocedural training can be conducted virtually with well-designed e-learning. However, educators must be aware of the limitation of e-learning in procedural skill acquisition. Psychomotor tasks may not be achieved with e-learning and video demonstrations alone.

\section{Tables and Figures}

Table 1: Participant Demographics

\begin{tabular}{|l|r|}
\hline \multicolumn{1}{|c|}{ Characteristics } & Frequency, (\%) \\
\hline Gender & $11(39.3)$ \\
\hline Male & $17(60.7)$ \\
\hline Female & $29(27-31)$ \\
\hline Age, median (IQR) & $27(93.1)$ \\
\hline Number of years working in polyclinic & $2(6.8)$ \\
\hline $1-2$ & \\
\hline $3-4$ & $26(89.7)$ \\
\hline Number of intrauterine contraceptive device insertions performed previously & $(3.4)$ \\
\hline 0 & 1 \\
\hline $1^{*}$ & $(3.4)$ \\
\hline $2^{*}$ & $(3.4)$ \\
\hline $3^{*}$ & 1 \\
\hline
\end{tabular}

Abbreviation: IQR, interquartile range.

*Participants had performed intrauterine contraceptive device insertions previously but not a Nova T device. 
Figure 1: Knowledge Test Scores Pre- and Post-E-Learning

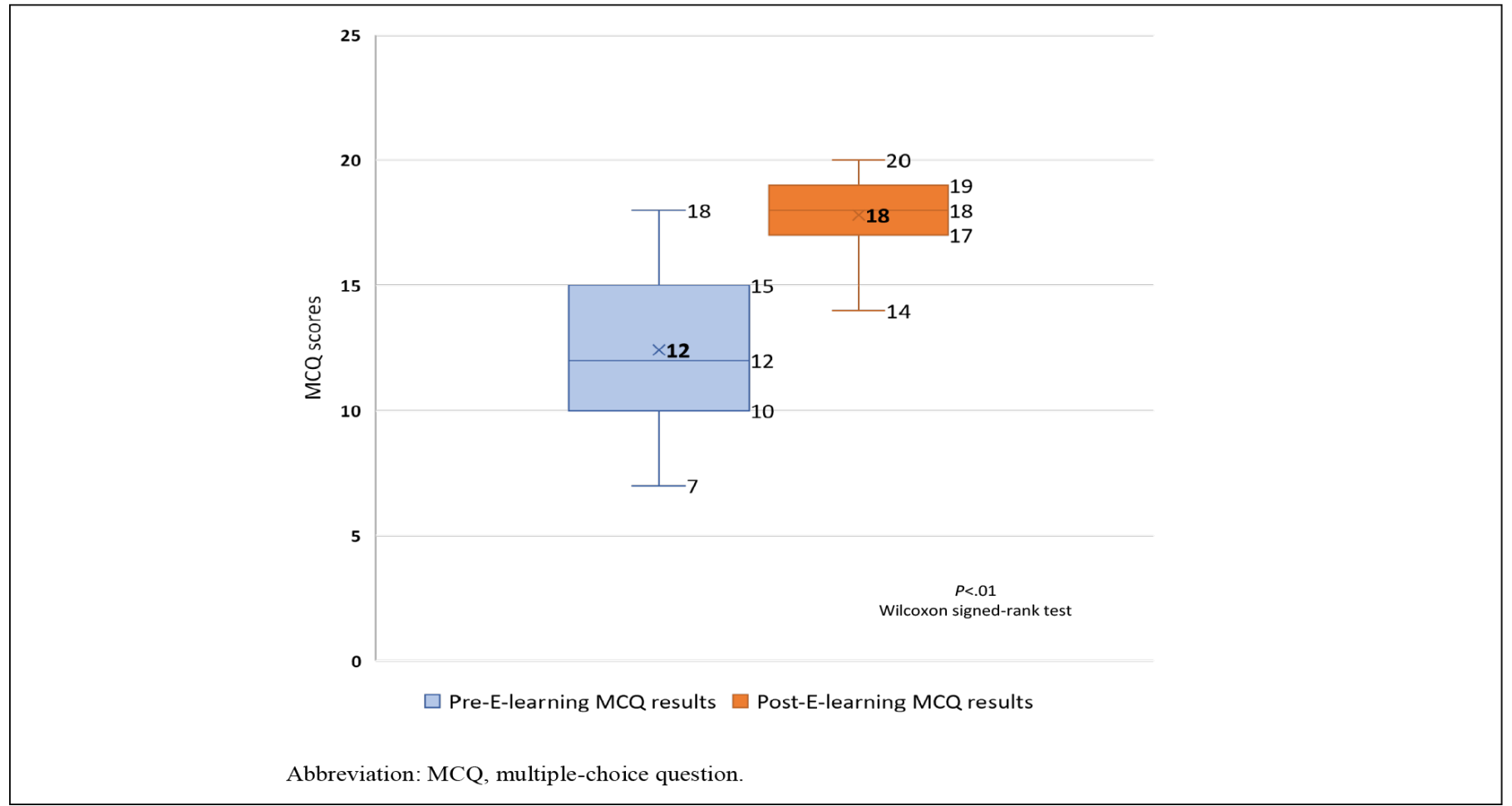

Figure 2: Procedural Step Skill Test Post-E-Learning 
Figure 3: Questionnaire Survey-Overall Satisfaction Level

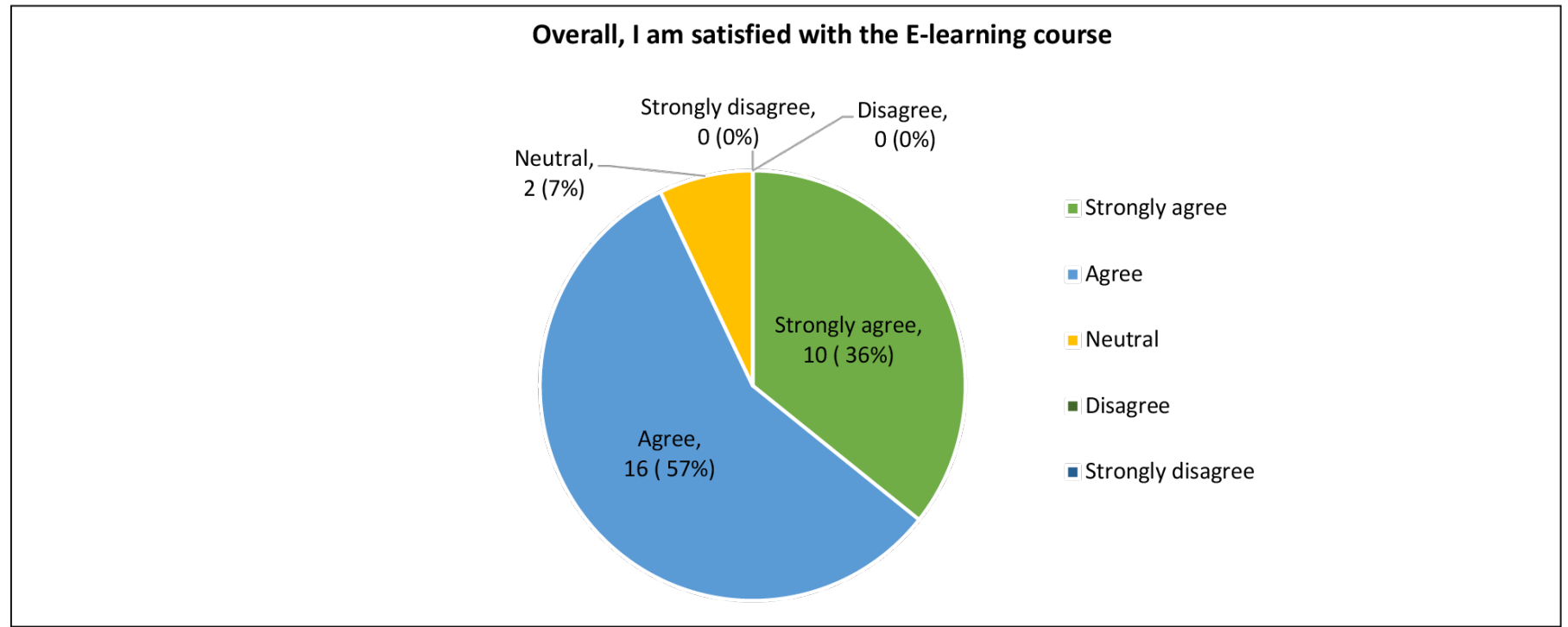

\section{Acknowledgments}

Financial Support: Financial support for this project was provided by a 2015 Academic Medicine Education Institute (AMEI) Education Grant awarded for the project title "Development and Assessment of E-learning Module for the Insertion of the Intrauterine Contraceptive Device."

\section{Presentation:}

1. IUCD insertion training-effectiveness of video assisted E-learning module. Short communication presented at Asia Pacific Medical Education Conference (APMEC) 2020. January 8-12, 2020, Singapore.

\section{Corresponding Author}

$\mathrm{Ng}$ Lai Peng, MBBS, MMed

Singhealth Polyclinics 167 Jalan Bukit Merah Connection One ( Tower 5), \#15-10, Singapore 150167. +65 62364800

ng.lai.peng@singhealth.com.sg

\section{Author Affiliations}

$\mathrm{Ng}$ Lai Peng, MBBS, MMed - SingHealth-Duke NUS Family Medicine Academic Clinical Programme, Singapore I Singhealth Polyclinics, Singapore

Eileen Koh Yi Ling, Bsc - Singhealth Polyclinics - Research, Singapore

\section{References}

1. Stoddard A, McNicholas C, Peipert JF. Efficacy and safety of long-acting reversible contraception. Drugs. 2011;71(8):969-980. doi:10.2165/11591290-000000000-00000

2. Ng LP, Koh EYL. Intrauterine contraceptive device insertion simulation training in primary care. Proc Singapore Healthc. 2018;27(4):229-233. doi:10.1177/2010105818757558

3. Garcia-Rodriguez JA, Donnon T. Using comprehensive video-module instruction as an Alternative Approach for Teaching IUD Insertion. Fam Med. 2016;48(1):15-20. Internet.

4. Ouyang M, Peng K, Botfield JR, McGeechan K. Intrauterine contraceptive device training and outcomes for healthcare providers in developed countries: a systematic review. PLoS One. 2019;14(7):e0219746. 
5. Herbitter C, Greenberg M, Fletcher J, Query C, Dalby J, Gold M. Family planning training in US family medicine residencies. Fam Med. 2011;43(8):574-581.

6. Garcia-Rodriguez JA. Filling the gaps between theory and daily clinical procedural skills training in family medicine. Educ Prim Care. 2016;27(3):172-176. doi:10.1080/14739879.2016.1169443

7. Touchie C, Humphrey-Murto S, Varpio L. Teaching and assessing procedural skills: a qualitative study. BMC Med Educ. 2013;13(1):69. doi:10.1186/1472-6920-13-69

8. Jang HW, Kim K-J. Use of online clinical videos for clinical skills training for medical students: benefits and challenges. BMC Med Educ. 2014;14(1):56. doi:10.1186/1472-6920-14-56

9. Pan M, Harcharik S, Moskalenko M, Luber A, Bernardo S, Levitt J. Instructional video for teaching venepuncture. Clin Teach. 2014;11(6):436-441. doi:10.1111/tct.12198

10. Maertens H, Madani A, Landry T, Vermassen F, Van Herzeele I, Aggarwal R. Systematic review of e-learning for surgical training. Br J Surg. 2016;103(11):1428-1437. doi:10.1002/bjs. 10236

11. Tarpada SP, Morris MT, Burton DA. E-learning in orthopedic surgery training: a systematic review. J Orthop. 2016;13(4):425-430. doi:10.1016/j.jor.2016.09.004

12. Botelho MG, Gao X, Jagannathan N. A qualitative analysis of students' perceptions of videos to support learning in a psychomotor skills course. Eur J Dent Educ. 2019;23(1):20-27. doi:10.1111/eje.12373

13. Zhang D, Zhou L, Briggs RO, Nunamaker JF Jr. Instructional video in e-learning: assessing the impact of interactive video on learning effectiveness. Inf Manage. 2006;43(1):15-27. doi:10.1016/j.im.2005.01.004

14. Mayer RE. Multimedia Principle. In: Multimedia Learning. 2nd ed. Cambridge: Cambridge University Press; 2009:223-241. doi:10.1017/CB09780511811678.017

15. Sun P-C, Tsai RJ, Finger G, Chen Y-Y, Yeh D. What drives a successful e-learning? An empirical investigation of the critical factors influencing learner satisfaction. Comput Educ. 2008;50(4):1183-1202. doi:10.1016/j.compedu.2006.11.007

16. Grantcharov TP, Reznick RK. Teaching procedural skills. BMJ. 2008;336(7653):1129-1131. doi:10.1136/bmj.39517.686956.47

17. Conti J, Shaw K. Update on long-acting reversible methods. Curr Opin Obstet Gynecol. 2015;27(6):471-475.

18. World Health Organization. Selected Practice Recommendations for Contraceptive Use. 3rd ed. Geneva, Switzerland: WHO; 2016.

19. Faculty of Sexual and Reproductive Healthcare. UK Medical Eligibility Criteria, 2016. https://www.fsrh.org /ukmec/. Accessed January 22, 2021.

20. LP Ng. Ma ZKL. Intrauterine Contraceptive device insertion E-learning questionaire survey. STFM Resource Library. https://resourcelibrary.stfm.org/HigherLogic/System /DownloadDocumentFile. ashx?DocumentFileKey=479f7860-dfcf-c75f-8c23-8a5c559b7988\& forceDialog=0. Accessed January 22, 2021.

21. LP Ng. Ma ZKL. Intrauterine Contraceptive device insertion E-learning, single best multiple choice questions. STFM Resource Library. https://resourcelibrary.stfm.org/HigherLogic/System /DownloadDocumentFile.ashx?DocumentFileKey=df0c353a-e3c1-d735-9515-181428ee7476\& forceDialog=0. Accessed January 22, 2021.

22. LP Ng. Ma ZKL. Intrauterine Contraceptive device insertion E-learning, procedural step checklist. STFM Resource Library. https://resourcelibrary.stfm.org/HigherLogic/System /DownloadDocumentFile.ashx?DocumentFileKey=ea75aeb2-33cf-f46d-24a9-06b0e6798df9\& forceDialog=0. Accessed January 22, 2021.

23. Yudkowsky R, Park YS, Lineberry M, Knox A, Ritter EM. Setting mastery learning standards. Acad Med. 2015;90(11):1495-1500. doi:10.1097/ACM.0000000000000887

24. McKinley DW, Norcini JJ. How to set standards on performance-based examinations: AMEE Guide No. 85. Med Teach. 2014;36(2):97-110. doi:10.3109/0142159X.2013.853119

25. Barsuk JH, Cohen ER, Wayne DB, McGaghie WC, Yudkowsky R. A Comparison of Approaches for Mastery 
Learning Standard Setting. Acad Med. 2018;93(7):1079-1084. doi:10.1097/ACM.0000000000002182

26. Kovacs G. Procedural skills in medicine: linking theory to practice. J Emerg Med. 1997;15(3):387-391. doi:10.1016/S0736-4679(97)00019-X

27. Huang GC, McSparron JI, Balk EM, et al. Procedural instruction in invasive bedside procedures: a systematic review and meta-analysis of effective teaching approaches. BMJ Quality \& Safety 2016;25:281-294. doi:10.1136/bmjqs-2014-003518

Copyright $(2021$ by the Society of Teachers of Family Medicine 